\title{
Variations in contact patterns and dispatch guideline adherence between Norwegian emergency medical communication centres - a cross-sectional study
}

Eirin N Ellensen ${ }^{1,2^{*}}$, Steinar Hunskaar ${ }^{2,3}$, Torben Wisborg ${ }^{4,5}$ and Erik Zakariassen 1,2,3

\begin{abstract}
Background: The 19 Norwegian Emergency medical communication centres (EMCCS) use Norwegian Index for medical emergency assistance (Index) as dispatch guidelines. Little is known about the use of Index, nor its validity. We aimed to document the epidemiology of contacts made to the public emergency medical phone number and the operators' self-reported use of Index as a first step towards a validation study.

Methods: We registered all medical emergency calls to the EMCCs during a $72 \mathrm{~h}$ period in a national cross sectional study. We subsequently sent a questionnaire to all EMCC operators in Norway, asking how they use Index. A combined outcome variable "use of Index" was computed through a Likert scale, range $1-5$. Regression models were used to examine factors influencing use.

Results: 2298 contacts were included. National contact rate was 56/1 000 inhabitants per year, range between EMCCs 34 - 119. Acute contact (life-threatening situations) rate was 21/1 000 per year, range between EMCCs 5 - 31. Index criteria 6 - 'Unresolved problem' accounts for $20 \%$ of the 113 contacts, range between EMCCs $10-42 \%$. The mean use of Index was 3.95 (SD 0.39), corresponding to "more than 75\% of emergency calls". There were differences in use of Index on EMCC level, range 3.7 - 4.4, and a multi regression model explained $23.4 \%$ of the variation in use. Operators working rotation with ground ambulance services reported reduced use of Index compared to operators not working in rotation, while distinct EMCC focus on Index increased use of Index compared to EMCCs with no focus on Index. Use of electronic records and operators experience were the main reasons given for not using Index.

Conclusions: There is a large variation between the EMCCs with regard to both contact patterns and use of Index. There is a relatively high overall self-reported use of Index by the operators, with variations on both individual and EMCC level.
\end{abstract}

Keywords: Criteria based dispatch, Emergency medical dispatch, Emergency medical services, Emergency medical communication systems, Triage, Guideline adherence

\footnotetext{
* Correspondence: eirin.nybo.ellensen@norskluftambulanse.no

'Department of Research, Norwegian Air Ambulance Foundation, PB 94,

Drøbak 1441, Norway

2Department of Global Public Health and Primary Care, University of Bergen

5050, Bergen, PB 7800, Norway

Full list of author information is available at the end of the article
} 


\section{Introduction}

Norway has a dedicated medical emergency phone number 113, which is answered and handled by an operator at the nearest Emergency medical communication centre (EMCC). The EMCCs dispatch the ambulance fleet, consisting of cars, boats, helicopters and planes, and facilitates radio and telephone communication amongst all the different participants in the chain of pre-hospital emergency medical care, from ambulance personnel through general practitioners on call to in-hospital specialists, and with other emergency authorities like police and fire brigades [1].

Norwegian Index for Medical Emergency Assistance (Index) [2] is the dispatch guidelines used by the EMCCs. It was developed from the Criteria Based Dispatch (CBD) guidelines in King County, Washington, USA [3], and introduced in Norway in 1994. Today Index is implemented as the only dispatch tool in all 19 EMCCs, serving an important role in securing equal high quality of the emergency health communication system throughout Norway $[2,4,5]$, as well as assisting the individual operator when handling an emergency call. Despite its importance on both the individual level (the patient in acute need of medical assistance) and on the systemic level (adequate resource allocation), Index has not been evaluated or validated during its nearly twenty years in use.

The CBD guidelines, and thus the Index, use symptom criteria to determine the urgency of the medical condition, and the appropriate level of response. This allows for a dynamic approach to the dispatch situation, compared to the more strict protocol compliance required by algorithm based dispatch systems. CBD and Index hence require an operator with a certain level of medical education and experience. In Norway, this is normally a registered nurse with experience from an emergency ward or an intensive care unit. The interpretation and modification of the formal guidelines according to individual skills will influence the criteria set, introduce an unknown variance in guideline adherence and hence lower the validity of any study regarding dispatch [6]. Guideline adherence is defined as "conformity in fulfilling or following official, recognized, or institutional requirements, guidelines, recommendations, protocols, pathways, or other standards" [7]. The algorithm based dispatch systems, such as Medical Priority Dispatch System (MPDS), traditionally use the term 'compliance' when referring to protocol adherence [6,8,9]. MPDS monitor compliance frequently along with many other quality markers [9], facilitating validity studies.

To our knowledge there are no international studies addressing CBD adherence. A recent systematic review on adherence to guidelines and protocols in the prehospital setting was not able to identify any eligible study in the emergency medical dispatch setting, neither CBD nor MPDS based [10]. However, a Norwegian study from 2005 evaluated dispatch in drug-related emergencies, and found an Index adherence of $99 \%$ based on multiple choice questionnaire, but only $64 \%$ guideline adherence based on log recordings [11].

A study on the epidemiology of medical emergency contacts made to three Norwegian EMCCs during a three month period in 2007 [12], focused on acute life threatening situations coded as acute only. The study showed statistically significant differences in the acute response rates among the EMCCs, but gave no explanation with regards to whether these differences could indicate variations in EMCC triaging or differences in the populations. National data on different urgency levels, use of Index criteria codes and contact rates to explore possible variations on a national level or study the triaging component further was not available in 2011 when we started the present study, but is now available as an annual report [13].

Before starting a validation study we needed to document the epidemiology of the contacts made to 113 and the emergency medical dispatch guideline adherence. The first aim of this study was to document possible differences in urgency levels, Index criteria and contact rates among the EMCCs. The second aim was to document the operators' self-reported use of Index by a questionnaire, and determine factors influencing the use.

\section{Methods and materials \\ Setting}

Autumn 2011 there were 19 EMCCs in Norway, belonging to four regional health trusts. The EMCCs differ in size, covering from 65000 to 1165000 inhabitants, with nearly 5 million inhabitants in total $[14,15]$. They are traditionally manned by registered nurse operators who answer and handle the 113 calls, and ambulance educated resource coordinators who dispatch and coordinate the ambulance fleet.

In addition to using the same dispatch guideline to prioritize and handle contacts [2], all EMCCs use the same software program Acute medical information system (AMIS) to register information and document each contact. AMIS contains information on Index criteria used, urgency level, and response dispatched.

\section{Epidemiology - cross sectional population based survey}

All 19 EMCCs contributed with data from every medical emergency contact made to the emergency medical phone number 113, during a 72 hour set period in August 2011. The data were collected in form of AMIS printouts, and contained information about date, time, 
the caller role, the patients sex and age, Index criteria used, urgency, response, and involvement of ambulances, air ambulances, GPs and others for each contact. Only calls registered to the 113 telephone line was included, excluding calls made by direct lines from fire brigades, police and Local emergency communication centers (LEMCs). Duplicates, maculated incidents and misdialing were also excluded (Figure 1).

\section{Norwegian index for medical emergency assistance (index)}

Index is a flip-over in large format consisting of a Start page, 36 symptom cards and 4 administrative cards. The Start page is built like an algorithm and clarifies important information as location, phone number, vital functions and a brief questioning on the patient's problem. The operator then moves on to the proper symptom card, determined by the problem presented. The symptom cards display a list of different criteria in decreasing severity, from the acute symptoms at the top and all the way down to the non-urgent symptoms at the bottom. The operator starts at the top and moves downwards through specific criteria, until one criterion is finally met. This criterion then determines the response dispatched. Index divides the response into three categories; red, yellow and green. Red response is when the situation is acute and life threatening, and calls for immediate "blue lights and sirens" ambulance dispatch and GP on-call alarm. Yellow response is an urgent and potentially life-threatening situation where ambulance and GP alarm is constantly assessed. Green responses are non-urgent situations, where the patients often are referred to the primary level of emergency care, unless there is an obvious need of ambulance transport.
Self-reported use of index - questionnaire based survey There were no validated questionnaires available to address our aim, so we developed a questionnaire in cooperation with one of the EMCCs and tested it on operators there to avoid possible misunderstandings.

Questions on their use of Index were arranged as a Likert scale of five symmetric response formats; never, seldom $(<25 \%)$, sometimes, often $(>75 \%)$ and always. The eleven different questions were all expressed in the same form: "During a real emergency call, how often do you...?" and covered different aspects of Index (Table 1). In addition to asking directly about use of Start page, we also asked specific questions from the Start page, like "... clarify if the patient is awake?" and "... if the patient can talk?" to unmask actual use of Start page and not just flipping of the page. The operators were asked about their educational level, EMCC work experience, initial training and later repetitions with regards to use of Index. We also asked if they thought there was focus on use of Index at their EMCC. The operators were given the possibility to add explanations or points of view at the end of the questionnaire. The questionnaire was distributed in paper through EMCC management, but returned directly to the research facility to secure anonymity for the individual operator.

\section{Population}

There were 429 persons working as operators in the EMCCs when our study started. An operator was defined as the person for whom the primary occupation is to answer the emergency medical phone call. A resource coordinator was defined as a person for whom the primary occupation is to coordinate the ambulance fleet.

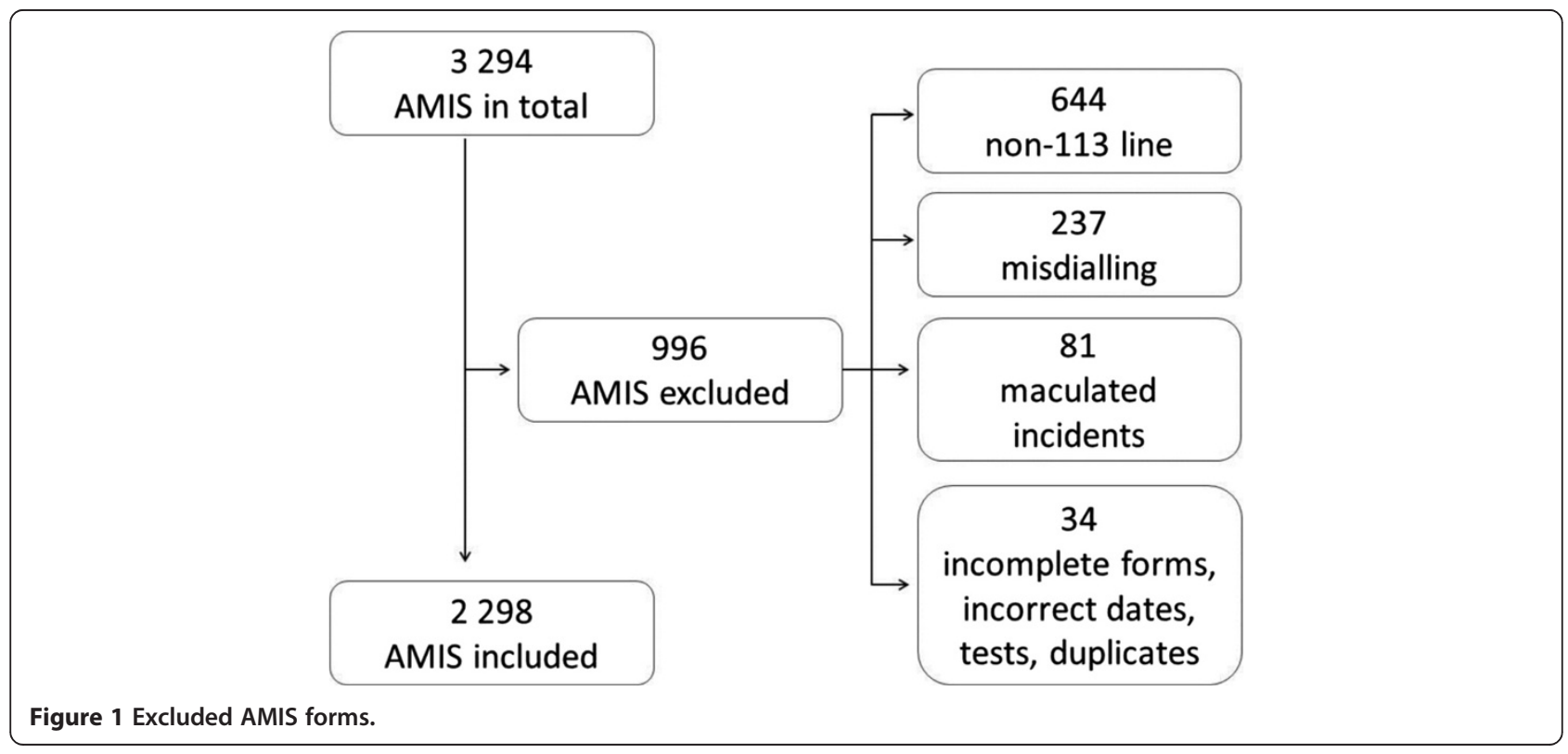


Table 1 Questions used to determine use of Index

\begin{tabular}{|c|c|c|c|}
\hline & $\mathbf{N}$ & Mean & SD \\
\hline \multicolumn{4}{|l|}{ When receiving a true emergency medical call, please specify how often you do the following: } \\
\hline I use the table format Index during the conversation & 270 & 4.02 & 0.82 \\
\hline I use Start page & 272 & 3.36 & 1.23 \\
\hline I clarify if the patient is awake & 270 & 4.93 & 0.26 \\
\hline I clarify whether the patient can talk or not & 270 & 4.32 & 0.73 \\
\hline I do not decide upon urgency before criteria (reversed in questionnaire) & 272 & 3.02 & 0.87 \\
\hline I do not determine criteria code without using Index (reversed in questionnaire) & 269 & 3.80 & 0.94 \\
\hline I start on top of the symptom card and work my way downwards & 267 & 4.01 & 0.86 \\
\hline I ask further questions & 269 & 4.09 & 0.74 \\
\hline I give advices to the caller/patient, if needed & 270 & 4.56 & 0.56 \\
\hline I give advices to health personnel on scene, if needed & 268 & 3.70 & 1.00 \\
\hline If Index is not used during the call, I check afterwards whether I have covered everything & 263 & 3.63 & 1.02 \\
\hline Total (Likert score) & & 3.95 & 0.39 \\
\hline
\end{tabular}

$1=$ Never, $2=$ Seldom $(<25 \%), 3=$ Sometimes, $4=$ Often $(>75 \%), 5=$ Always. $N=272$ operators.

Only questionnaires from operators were included in the study.

\section{Statistical analysis and ethical approvals}

Continuous data are presented as mean (SD) or median (quartiles) for symmetric and skewed data, respectively. Rates are calculated as contacts per 1000 inhabitants per year. The five response formats on the Likert scale were valued 1 to 5 , and the outcome variable "use of Index" was calculated for each operator as the mean score from all eleven questions constituting the Likert scale. Q-Q plots were used to check the outcome variable for normal distribution. We explored the variation in mean use of Index with respect to each of the explanatory variables by one-way analysis of variance (ANOVA) and univariate linear regression. All explanatory variables were then entered in a full multiple linear regression model. Effect estimates are reported with 95\% confidence intervals (CI). A possible hierarchical effect of EMCC central was assessed by adjusting for central in a mixed model. There were only marginal differences in effect estimates when adjusting for the mixed effect, and we therefore consequently report results from the ANOVA and linear regression models.

The statistical analyses were performed using Statistical Package for the Social Sciences (IBM SPSS Statistics 20) and STATA (Stata/IC 12.1). P-values below 0.05 were considered statistically significant.

Both studies were evaluated by the Regional committees for medical and health research ethics and considered not to be in need of ethical assessment. Exemption for consent was given by the Regional committees for medical and health research ethics in terms of receiving and analyzing sensitive data. The Data Protection Official for Research approved the study.

\section{Results} Epidemiology

We collected 3294 AMIS forms during a 72 hour period, of which 2298 were included for analyses. This gives a calculated overall national emergency medical contact rate of 56/1 000 inhabitants per year $(95 \% \mathrm{CI}$ 54-59), ranging from 34 (95\% CI 22-46) to 119 (95\% CI 91-146) among the different EMCCs. Of the 2298 contacts $37 \%$ were assessed to be acute, $34 \%$ urgent, and $27 \%$ non-urgent, while $1.8 \%$ of the contacts had no urgency assessment. Looking at acute contacts separately, the rates varied significantly from 5 to $31 / 1000$ inhabitants per year (Table 2).

The most frequently used Index criteria was " 6 - Unresolved issue" (Table 3), which accounted for $20 \%$ of all contacts. The use of this criteria differed from $10 \%$ at one EMCC to $42 \%$ at another. The use of other criteria like "5 - Ordered assignment" and "28 - Psychiatry Suicide" also varied much between different EMCCs, from 0 to $22 \%$ and $19 \%$ respectively (Table 3 ). The urgency assessments on criteria 6 differed between the EMCCs; acute $0-53 \%$, urgent $0-67 \%$ and non-urgent $9-86 \%$ (Figure 2).

\section{Use of Index}

We received 275 questionnaires after three reminders, of which 272 questionnaires were included in the study, representing all 19 EMCCs (response rate 63.4\%). The operators were mainly female, registered nurses, of whom many worked in rotation at either emergency rooms (ER) or ambulances (Table 4). The majority received training in use of Index when starting to work at the EMCC, but later repetitions were rare (Table 4). The EMCC work experience ranged from 1 month to 36 years, with a median (quartile) time of $6(3,12)$ years. 
Table 2 Acute contact rates between different Emergency medical communication centres

\begin{tabular}{|c|c|c|c|}
\hline \multirow[t]{2}{*}{ EMCC } & Acute contacts & Acute contacts & $95 \% \mathrm{Cl}$ \\
\hline & $\mathbf{N}$ & Rate & Rate \\
\hline 1 & 19 & 31 & $(17-46)$ \\
\hline 2 & 14 & 14 & $(7-22)$ \\
\hline 3 & 11 & 21 & (8-33) \\
\hline 4 & 13 & 12 & $(5-18)$ \\
\hline 5 & 3 & 5 & $(0-10)$ \\
\hline 6 & 17 & 15 & $(8-22)$ \\
\hline 7 & 46 & 19 & $(13-24)$ \\
\hline 8 & 12 & 13 & $(6-21)$ \\
\hline 9 & 18 & 15 & $(8-22)$ \\
\hline 10 & 12 & 14 & $(6-21)$ \\
\hline 11 & 87 & 25 & $(20-31)$ \\
\hline 12 & 23 & 16 & $(10-23)$ \\
\hline 13 & 34 & 12 & $(8-16)$ \\
\hline 14 & 56 & 24 & $(17-30)$ \\
\hline 15 & 78 & 26 & $(20-32)$ \\
\hline 16 & 38 & 15 & $(11-20)$ \\
\hline 17 & 242 & 25 & $(22-28)$ \\
\hline 18 & 76 & 24 & $(19-30)$ \\
\hline 19 & 42 & 18 & $(13-24)$ \\
\hline Total & 841 & 21 & $(19-22)$ \\
\hline
\end{tabular}

Contacts made to all the EMCCs, and assessed by the operators to be of the most urgent of three urgency categories. $\mathrm{N}=$ number of acute contacts during a data collection period of $72 \mathrm{~h}$. Rates calculated per 1000 inhabitants per year.

Use of Index ranged from 2.91 to 4.82 , with overall mean (SD) for all operators being 3.95 (0.39), corresponding closely to the response format "often, $>75 \%$ " $(=4)$ in the questionnaire. The individual questions on use of Index ranged from $4.93(0.26)$ for "I clarify if the patient is awake" to 3.02 (0.87) for "I do not decide upon urgency before criteria" (Table 1). ANOVA showed significant differences in use of Index among the EMCCs $(\mathrm{p}<0.001)$, with the EMCCs ranging from $3.7(0.24)$ to $4.4(0.39)$. Figure 3 shows the spread between the EMCCs. Possible explanatory variables for use of Index were analyzed by univariate and multiple linear regression models (Table 5). Time, the effect of rotation with ambulance and a clear focus on use of Index at workplace on use of Index were statistically significant, with focus being by far the strongest component $(\beta=0.46, \mathrm{p}<0.001)$ (Table 5$)$.

For use of Start page separately, we found that $47 \%$ reported using the Start page often or always (> 75\% of real emergency calls), while $93 \%$ always checked if the patient was awake and $46 \%$ if he could talk. The reasons given for not using Index are shown in Table 6.

The question "work percentage at EMCC" was misinterpreted by many operators with regard to whether include rotatory work at ER/ambulances or not, and was hence excluded from analysis. Explanations offered in free text did not add any relevant information on the questions addressed, and were not analyzed.

\section{Discussion}

The main finding of the epidemiological part of the study was the wide spread between the different EMCCs, regarding both overall contact rates, specific acute rates and use of different Index criteria when assessing the situations. The questionnaire study showed a relatively high overall use of Index reported by the operators. It also showed large unexplained variations in the use of Start page and several symptom cards.

\section{Strengths and weaknesses of the studies}

The epidemiology study brings new knowledge about contacts made to 113, while the self-reported use of Index represents the first step towards determining use of Index. Both studies include all 19 EMCCs and hence are representative on a national level.

The short study period of $72 \mathrm{~h}$ limits the validity of extrapolation, and was mainly due to capacity issues in both ends, as data had to be exchanged in paper format at the time being. The questionnaire was not validated, as we wanted the main study population as large as possible, in terms of both individuals and centers.

Self-reporting allows for over- and underestimation. This was a calculated risk as we aimed to document how the operators themselves thought they used Index, and the results must be interpreted as subjective. We will address the problem with subjective contra objective use of Index in future audio-log study. The response rate of $63.4 \%$ could represent a selection bias, but the large range in 'use of Index' score, both on an individual level and on EMCC level, together with all EMCCs being represented, indicates that we have a representative material. The response rate can to some extent be explained by all information, distribution and reminders depending on the management at each EMCC. The official policy by the EMCC managements on always using Index, combined with the questionnaires and reminders being provided by the management, raises the possibilities of an "eagerness to please" bias. This was attempted opposed by ensuring anonymity and allowing the filled out questionnaires to return directly to the main investigator, through prepaid preaddressed envelopes following each single questionnaire. Recall biases are likely to occur when trying to recall what one usually does in certain situations. The use of a non-validated questionnaire led to misinterpretation of some questions. Never and always are narrow categories, and the results on use of Index might have been more correct if the categories had been more evenly distributed. 
Table 3 Top ten Index criteria

\begin{tabular}{|c|c|c|c|c|c|c|c|c|c|c|c|c|c|c|}
\hline \multirow{3}{*}{ EMCC } & \multirow[b]{2}{*}{ Contacts } & \multicolumn{13}{|c|}{ Criteria number* } \\
\hline & & 6 & 10 & 5 & 30 & 33 & 29 & 35 & 25 & 28 & 27 & All other & Missing & Total \\
\hline & $\mathbf{N}$ & $\%$ & $\%$ & $\%$ & $\%$ & $\%$ & $\%$ & $\%$ & $\%$ & $\%$ & $\%$ & $\%$ & $\%$ & $\%$ \\
\hline 1 & 72 & 14 & 3 & 3 & 6 & 4 & 6 & 4 & 4 & 12 & 9 & 13 & 25 & 100 \\
\hline 2 & 44 & 20 & 9 & 5 & 0 & 9 & 7 & 5 & 5 & 5 & 5 & 20 & 11 & 100 \\
\hline 3 & 40 & 22 & 8 & 8 & 0 & 14 & 0 & 11 & 8 & 8 & 3 & 8 & 18 & 100 \\
\hline 4 & 56 & 32 & 11 & 4 & 11 & 11 & 5 & 5 & 2 & 2 & 2 & 14 & 2 & 100 \\
\hline 5 & 32 & 27 & 13 & 0 & 3 & 7 & 7 & 0 & 0 & 13 & 3 & 19 & 13 & 100 \\
\hline 6 & 53 & 19 & 6 & 6 & 6 & 2 & 6 & 4 & 4 & 19 & 4 & 19 & 8 & 100 \\
\hline 7 & 89 & 26 & 6 & 7 & 2 & 4 & 6 & 11 & 11 & 0 & 4 & 22 & 0 & 100 \\
\hline 8 & 46 & 15 & 7 & 11 & 4 & 2 & 9 & 4 & 9 & 15 & 9 & 9 & 7 & 100 \\
\hline 9 & 67 & 22 & 5 & 3 & 2 & 2 & 0 & 5 & 5 & 10 & 3 & 15 & 33 & 100 \\
\hline 10 & 30 & 30 & 10 & 10 & 10 & 7 & 3 & 0 & 3 & 0 & 10 & 17 & 0 & 100 \\
\hline 11 & 240 & 10 & 10 & 4 & 10 & 5 & 4 & 4 & 3 & 1 & 4 & 15 & 30 & 100 \\
\hline 12 & 65 & 25 & 2 & 6 & 11 & 10 & 3 & 5 & 5 & 10 & 0 & 25 & 2 & 100 \\
\hline 13 & 117 & 30 & 13 & 2 & 11 & 7 & 10 & 4 & 4 & 4 & 6 & 12 & 1 & 100 \\
\hline 14 & 114 & 14 & 7 & 8 & 4 & 4 & 4 & 4 & 7 & 5 & 5 & 20 & 18 & 100 \\
\hline 15 & 168 & 23 & 14 & 8 & 5 & 5 & 7 & 7 & 7 & 4 & 7 & 13 & 1 & 100 \\
\hline 16 & 119 & 31 & 8 & 10 & 5 & 5 & 3 & 6 & 4 & 6 & 4 & 18 & 0 & 100 \\
\hline 17 & 605 & 14 & 10 & 4 & 6 & 6 & 7 & 5 & 5 & 3 & 5 & 18 & 19 & 100 \\
\hline 18 & 228 & 23 & 10 & 22 & 4 & 4 & 4 & 6 & 4 & 5 & 0 & 18 & 0 & 100 \\
\hline 19 & 113 & 42 & 10 & 4 & 3 & 7 & 5 & 3 & 6 & 6 & 3 & 12 & 0 & 100 \\
\hline Total & 2298 & 20 & 9 & 7 & 6 & 6 & 5 & 5 & 5 & 5 & 5 & 16 & 12 & 100 \\
\hline
\end{tabular}

The most frequently used criteria in Index (\%), for each of the 19 EMCC. All degrees of urgency included. $\mathrm{N}=$ total number of contacts during the study period of $72 \mathrm{~h}$. ${ }^{*}$ Criteria number explanation: 5 - Commissioned assignments (by health personnel), 6 - Unresolved issue, 10 - Chest pain, heart disease, 25 - Stomach pain, back pain, 27 - Reduced consciousness, paralyses, 28 - Psychiatry, suicide, 29 - Breathing issues, 30 - Intoxication, overdose, 33 - Wounds, fractures, minor injuries, 35 - Accidents.

\section{Epidemiology}

There is a wide spread in annual contact rates among the EMCCs, from 34 to $119 / 1000$ inhabitants. We have no national data on acute disease distribution to compare with, but one would not expect a similar spread in distribution. Accidents are shown to disperse a geographical pattern, a study from 2013 comparing a rural county with an urban/rural county found a higher accident rate in urban areas, but a higher mortality rate per accident in the rural areas [16]. This dispersion of accidents

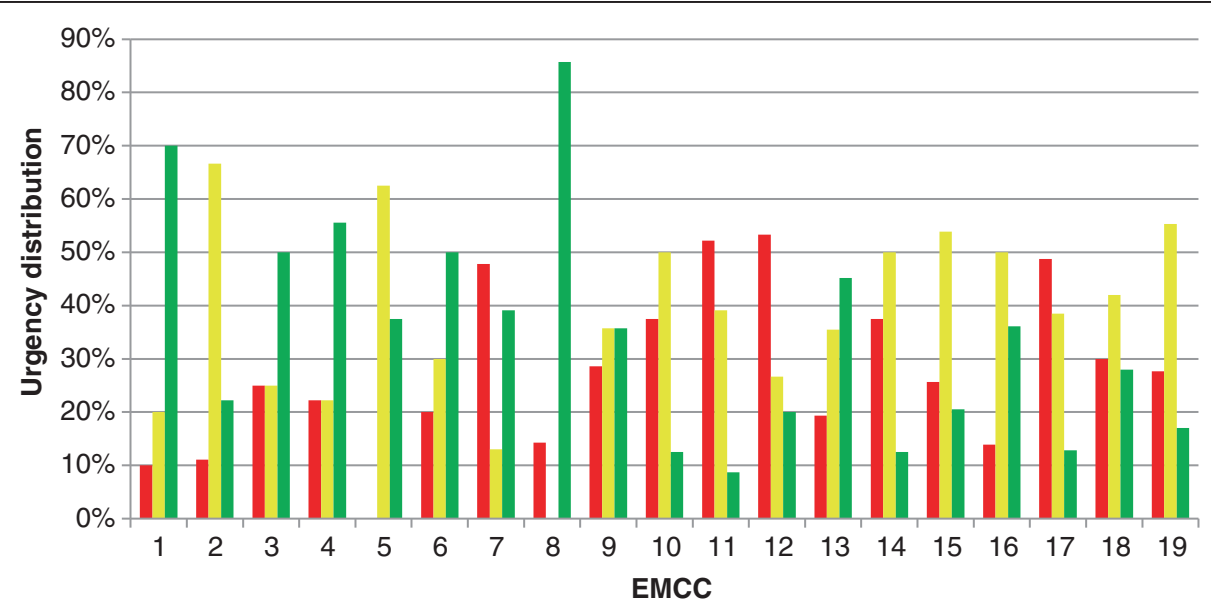

Figure 2 Use of Index criteria "6 - Unresolved issue" between the EMCCs. Differences in urgency assessment, and hence use of Index criteria «6 - Unresolved issue» between the different EMCCs. Red = acute, yellow = urgent, green = non-urgent contacts. 
Table 4 Operator characteristics

\begin{tabular}{|c|c|c|c|}
\hline & & $\mathbf{N}$ & $\%$ \\
\hline \multirow[t]{4}{*}{ Sex } & Female & 178 & 65 \\
\hline & Male & 85 & 32 \\
\hline & Missing & 9 & 3 \\
\hline & & 272 & 100 \\
\hline \multirow[t]{5}{*}{ Education } & Ambulance worker & 50 & 18 \\
\hline & Registered nurse & 230 & 85 \\
\hline & Ambulance worker and nurse & 16 & 69 \\
\hline & Other & 11 & 4 \\
\hline & Further education & 86 & 32 \\
\hline \multirow[t]{6}{*}{ Work rotation } & None & 73 & 27 \\
\hline & With emergency room & 116 & 43 \\
\hline & With ambulance & 44 & 16 \\
\hline & Other & 19 & 7 \\
\hline & Missing & 20 & 7 \\
\hline & & 272 & 100 \\
\hline \multirow[t]{6}{*}{ Work experience } & $\leq 1$ year & 27 & 10 \\
\hline & $>1 \leq 5$ years & 95 & 35 \\
\hline & $>5 \leq 10$ years & 60 & 22 \\
\hline & $>10$ years & 78 & 29 \\
\hline & Missing & 12 & 4 \\
\hline & & 272 & 100 \\
\hline \multirow[t]{6}{*}{ Training in use of Index } & Formal training from the start & 234 & 86 \\
\hline & Formal training eventually & 16 & 6 \\
\hline & Taught by colleagues & 8 & 3 \\
\hline & None & 2 & 1 \\
\hline & Missing & 12 & 4 \\
\hline & & 272 & 100 \\
\hline \multirow[t]{5}{*}{ Repetition of use of Index } & Regularly & 51 & 19 \\
\hline & Irregularly & 129 & 47 \\
\hline & None & 71 & 26 \\
\hline & Missing & 21 & 8 \\
\hline & & 272 & 100 \\
\hline
\end{tabular}

Background information on the operators' $(\mathrm{N}=272)$ educational and work experience, together with experienced initial training in use of Index and later repetitions.

does not account for the wide spread in contact rates, and this could indicate that the population uses 113 differently depending on location. A previous study of geographic variations in alerting, dispatch and response found that while severity of illness/trauma had no effect on the use of 113, the use was lower in rural areas compared with urban areas [17]. This could be due to local differences in organisation of LEMCs and casualty clinics, and cultural differences for when to access the different levels of emergency health services.
When separating contacts assessed to be acute, we also find a large spread in rates between the different EMCCs. The overall acute contact rate of 21 is slightly lower than reported by a previous study based on data from three EMCCs. They found a three month acute rate of 6.2/1 000 inhabitants in 2007, giving an annual rate of 25/1 000 [18]. As our study shows that the EMCCs differ with regards to both urgency distributions and contact rates, differences in inclusion of EMCCs will affect these outcomes.

A recently published study from Denmark studied Danish Index' ability to triage patients according to severity [19], following the implementation of the CBD guidelines in Denmark. They found a national acute (emergency level A) rate of 17 ambulance dispatches/ 1000 inhabitants per year, which is somewhat lower than our 21 acute contacts/1 000 inhabitants per year. There were also differences between their included areas: with 13, 17 and 21 acute ambulance dispatches/1 000 per year. One possible explanation could be the large differences in EMCC population size, with five centres in Denmark and 19 in Norway covering 5.5 and 5 million inhabitants respectively. This difference could influence use of Index, and hence incidence of acute criteria codes. Cultural differences in what emergency medical level to access and differences in pre-hospital emergency medicine organization could influence this.

On primary health care level, a study of 85000 contacts from 2007 investigated the distribution of urgency levels in the Norwegian emergency primary healthcare services: acute $2.3 \%$, urgent $21.1 \%$ and non-urgent $76.6 \%$ [20]. Compared to our findings (acute 37\%, urgent 35\%, non-urgent $26 \%$ ), these differences show that the population as a whole know what level to address, depending on degree of medical emergency.

The most frequently used Index criteria, all urgencies included, was "6 - Unresolved issue". This criterion covers a whole range of situations; from the unclear situations where the operator gets too little information to choose another criterion to well-defined situations where no other criteria match. The large differences between the EMCCs, both in terms of total use of this criterion and the variation in urgencies as it was used in, clearly indicates a variation in use of Index from center to center.

\section{Use of Index}

The operators reported a relatively high overall use of Index, corresponding to "in over $75 \%$ of real emergency calls". The variation among the operators was quite large though. Although we found some factors associated with positive and negative effects on use of Index, we were only able to explain $23.4 \%$ of this variation, indicating that there are factors influencing use of Index that we 


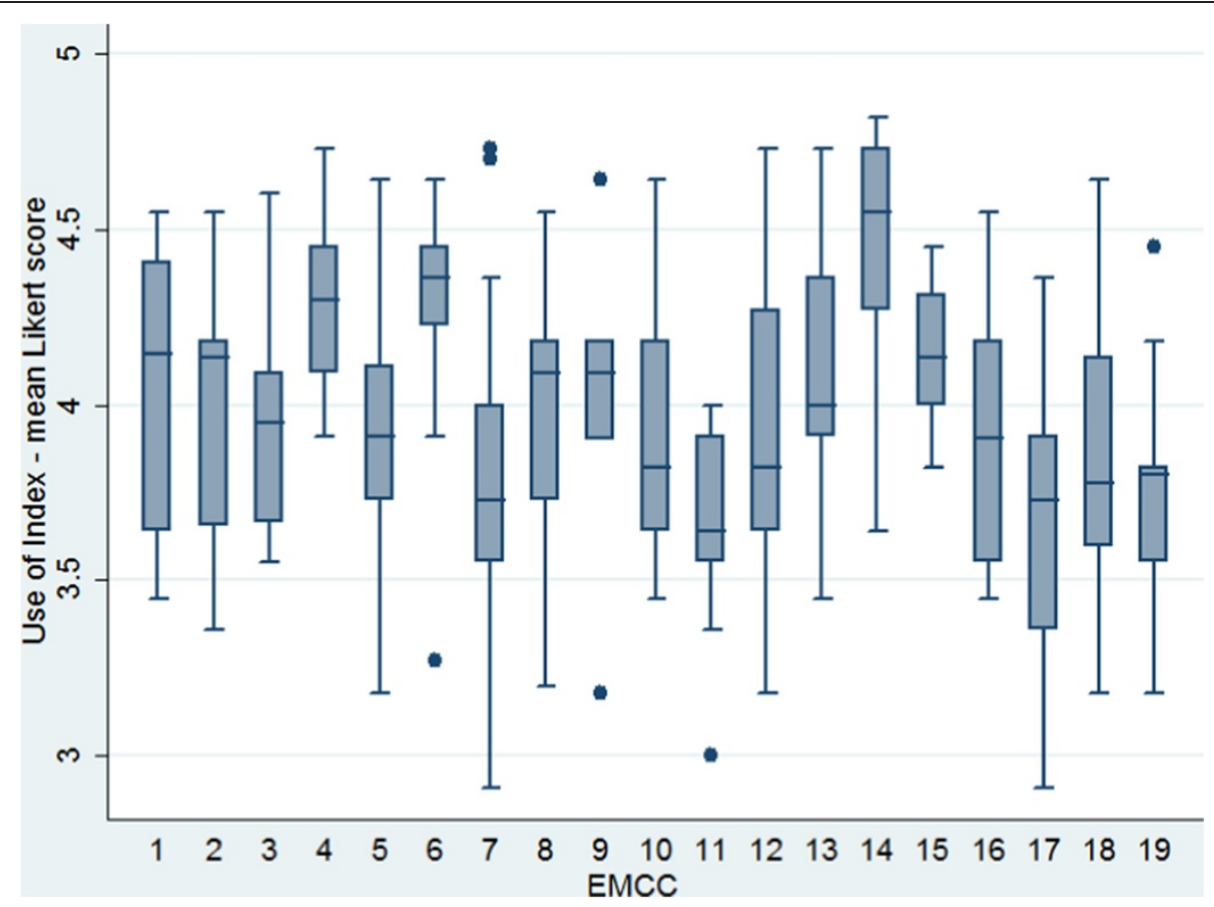

Figure 3 Use of Index between the EMCCs. Box plot of mean Likert-score values, displaying use of Index between the different EMCCs. The box represent the first, second (median) and third quartile of the data. The whiskers show the lowest datum within 1.5 interquartile range of the first quartile, and the highest datum within 1.5 interquartile range of the third quartile. Outliers are shown as dots.

were not able to uncover. The main reason given for not using Index was AMIS, and the confidence that this software program provided the necessary key words to properly assess the acute medical emergency situation. The operators' background was to great extent as expected. It was an experienced group of employees, with median working time at the EMCC of 6 years. The results indicated decent routines for training new operators in use of Index, but not for repetition.
Our result of approximately $75 \%$ use of Index lies between the divergent findings from the 2005 study, with 99\% self-reported Index guideline adherence and 64\% adherence based on log-recordings [11]. To our knowledge there are no other studies addressing adherence to criteria based guidelines. As algorithm based dispatch systems require a much stricter adherence to protocol they have systems developed to monitor and increase protocol compliance, among other quality markers [6,8,21-24].

Table 5 Explanatory variables for the variation in use of Index

\begin{tabular}{|c|c|c|c|c|c|c|}
\hline & \multicolumn{3}{|c|}{ Univariate linear regression } & \multicolumn{3}{|c|}{ Multiple linear regression } \\
\hline & Estimate & $(95 \% \mathrm{Cl})$ & P-value & Estimate & $(95 \% \mathrm{Cl})$ & P-value \\
\hline Working experience & -0.011 & $(-0.018,-0.003)$ & 0.004 & -0.012 & $(-0.019,-0.004)$ & 0.002 \\
\hline \multicolumn{7}{|l|}{ Rotation } \\
\hline With ground ambulance & -0.218 & $(-0.360,-0.076)$ & 0.003 & -0.213 & $(-0.348,-0.078)$ & 0.002 \\
\hline With emergency room & 0.054 & $(-0.057,0.165)$ & 0.337 & 0.033 & $(-0.074,0.139)$ & 0.545 \\
\hline With others & -0.093 & $(-0.283,0.098)$ & 0.339 & -0.046 & $(-0.229,0.137)$ & 0.621 \\
\hline \multicolumn{7}{|l|}{ Repetition of use of Index } \\
\hline Regularly & 0.214 & $(0.074,0.354)$ & 0.003 & 0.062 & $(-0.080,0.205)$ & 0.390 \\
\hline Irregularly & 0.090 & $(-0.023,0.203)$ & 0.118 & -0.002 & $(-0.116,0.111)$ & 0.969 \\
\hline \multicolumn{7}{|l|}{ Focus on use of Index } \\
\hline Clear focus & 0.522 & $(0.312,0.732)$ & $<0.001$ & 0.459 & $(0.240,0.679)$ & $<0.001$ \\
\hline Some focus & 0.293 & $(0.073,0.512)$ & 0.009 & 0.207 & $(-0.013,0.426)$ & 0.065 \\
\hline
\end{tabular}

Exploration of possible explanations for the variation in use of Index, by univariate and multiple linear regression models.

Working experience $=$ effect per year. P-value $<0.05$ was regarded as statistically significant. Explained variation, $R^{2}=0.234$ for the multiple linear regression model. 
Table 6 Reasons for not using Index or Start page

\begin{tabular}{lrr}
\hline & N & $\%$ \\
\hline Question: When not using the Index flip over, why? & 149 & 55 \\
I find the criteria in AMIS & 91 & 33 \\
I know it & 55 & 20 \\
It takes too long & 44 & 16 \\
It is cumbersome to move my hands between Index & & \\
and the keyboard & 24 & 9 \\
It has become a habit & 23 & 8 \\
I am expected to know it & 13 & 5 \\
It is of little help & 0 & 0 \\
I am not trained to use it & & \\
Question: When not using the Start page, why? & 117 & 43 \\
I know it & 108 & 40 \\
I prefer to go straight to proper card & 57 & 21 \\
I find what I need in AMIS & 38 & 14 \\
It is cumbersome to move the hands between flip & & \\
over and keyboard & 27 & 10 \\
It takes too long &
\end{tabular}

Given reasons for not using Index/Start page, checked off by the operators.

Total number of operators $=272$.

Although compliance is highlighted as such an important feature of MPDS, less than 3\% of the registered users are Accredited Centers of Excellence [25], which among other quality measurements include a minimum of $90-95 \%$ compliance with different parts of the protocol [8].

Given the purpose of CBD guidelines, to assist health care personnel in decision making rather than defining specific questions or actions to be taken [3], use of Index was not expected to reach full score. Looking at the different explanatory variables though, it was rather unexpected that time working at an EMCC had so little effect on use of Index. It was equally unexpected that the effect of what EMCC the operator works at was so negligible, as we held this to be the natural explanation for the variations in use of Index.

Working in rotation with ground ambulance is the variable strongest associated with decreased use of Index. This is not associated with educational level, as half the operators rotating with ambulance are registered nurses and there is no difference in use of Index between the different educational levels among those rotating with ambulance. Previous research has found that individual experience and professional autonomy affects guideline adherence [10]. Our study finds no effect on use of Index for operators rotating with ER. A Dutch study on protocol adherence among emergency and ambulance nurses found the opposite result; ambulance nurses are more likely to hold protocol over experience while emergency nurses are more likely to hold experience over protocol [26].
The low use of Start page is reflected in the reasons given for not using it. They know it, and find the key words they need in AMIS screen. The Start page $\left(3^{\text {rd }}\right.$ edition) includes the phrase: "Is the patient awake and can talk?" [2]. The low percentage reporting to actually ask about this latter, confirms that neither AMIS nor memory equals Start page, and one may speculate that the reason for including this question is not fully understood among those using the tool.

The main reason for not using Index appears to be that the operators choose to use the AMIS screen above Index, and a belief that AMIS provides the necessary keys to assist them in properly assessing the situation. This might be of some concern, since AMIS does not supply any support in neither decision making nor advices for the public or health personnel at scene, and hence cannot replace Index. Furthermore, discarding the guidelines in favor of memory and own experience is a potential hazard of losing vital information or getting sidetracked due to unstructured interrogation. This risk is strongly advocated by critics of criteria based dispatch $[9,22]$.

Based on our findings we would recommend increased focus on use of Index at each EMCC. The systematic review on guideline and protocol adherence in the prehospital and emergency care setting [10] finds that tailored strategies towards identified barriers improve professional practice, as well as strategies aimed at influencing factors improve guideline adherence. Evidence based recommendations and a relationship between guideline adherence and clinical outcome are also mentioned as important motivational factors for guideline adherence.

In this study we estimated a mean value for use of Index among operators in Norway, based on self-report, which obviously represents a subjective view. Investigating the real practice based on objective data is therefore a natural next step in documenting use of Index.

\section{Conclusion}

The EMCCs varies regarding both contact rates and use of Index. The operators report use of Index in $>75 \%$ of real emergency situations, but there are large individual differences. Clear focus on use of Index at the EMCC was the strongest predictor for increased use, and work rotation with ground ambulance services was associated with decreased use.

\section{Abbreviations}

AMIS: Acute medical information system; ANOVA: Analysis of variance; CBD: Criteria based dispatch; Cl: Confidence interval; EMCC: Emergency medical communication centre; ER: Emergency room; GP: General practitioners; LEMC: Local emergency medical communication centre; MPDS: Medical priority dispatch system; SD: Standard deviation; SPSS: Statistical Package for the Social Sciences.

\section{Competing interests}

The authors declare no competing interests. The funding foundation had no participation in conducting the study or writing the manuscript. 


\section{Authors' contributions}

All authors contributed in designing the studies. ENE collected and analysed the data, and drafted the manuscript. All authors contributed substantially in the interpretation of the findings and rewriting the manuscript. All authors read and approved this final version.

\section{Acknowledgements}

We would like to thank Jo Røislien at Norwegian Air Ambulance Foundation for excellent help on statistical issues. We also want to thank operators and management at all the 19 EMCCs in Norway for participating in the studies, and Tone Alfsvåg Engelsen at Bergen AMK for contributing in the planning process. The project was supported financially by the Norwegian ExtraFoundation for Health and Rehabilitation through EXTRA funds. Last but not least, we would like to thank all members of the Norwegian Air Ambulance Foundation for the financial support that makes this research possible.

\section{Author details}

Department of Research, Norwegian Air Ambulance Foundation, P.O. Box 94, N-1441, Drøbak, Norway. Department of Global Public Health and Primary Care, University of Bergen, P.O. Box 7804, N-5020 Bergen, Norway. ${ }^{3}$ National Centre for Emergency Primary Health Care, Uni Health, Uni Research, Kalfarv. 31, N-5018 Bergen, Norway. ${ }^{4}$ Anaesthesia and Critical Care Research Group, Faculty of Health Sciences, University of Tromsø, N-9037 Tromsø, Norway. ${ }^{5}$ Norwegian Trauma Competency Service, Oslo University Hospital, N-0450 Oslo, Norway.

Received: 22 August 2013 Accepted: 31 December 2013 Published: 8 January 2014

\section{References}

1. Norwegian Ministry of Health and Care services: Forskrift Om Krav til Akuttmedisinske Tjenester Utenfor sykehus. (Regulation on pre-hospital emergency medicine services) [http://www.lovdata.no/for/sf/ho/xo-20050318-0252.html]

2. Norwegian Medical Association: Norsk indeks for Medisinsk Nødhjelp (Noregian Index for Medical Emergency Assistance). 3rd edition. Stavanger: Laerdal Medical A/S; 2009.

3. Culley LL, Henwood DK, Clark JJ, Eisenberg MS, Horton C: Increasing the efficiency of emergency medical services by using criteria based dispatch. Ann Emerg Med 1994, 24:867-872.

4. Norwegian Medical Association: Medisinsk Nødmeldetjeneste, Standard for Prehospital Akuttmedisin. (Emergency health communication system) [http://www.kokom.no/hurtigvalg/nodmeldetjenesten.htm]

5. Norwegian Ministry of Health and Care Services: Stortingsmelding 43 (19992000) Om Akuttmedisinsk Beredskap. (About emergency preparedness) [http://www.regjeringen.no/Rpub/STM/19992000/043/PDFA STM199920000043000DDDPDFA.pdf]

6. Clawson JJ, Cady GA, Martin RL, Sinclair R: Effect of a comprehensive quality management process on compliance with protocol in an emergency medical dispatch center. Ann Emerg Med 1998, 32:578-584.

7. The National Centre for Biotechnology Information (NCBI): Medical Subject Headings (MeSH). [http://www.ncbi.nlm.nih.gov/mesh/?term=guideline+ adherence]

8. International Academies of Emergency Dispatch: Accreditation. [http://www. emergencydispatch.org/Accred20PointsMedical]

9. Clawson JJ, Martin RL, Hauert SA: Protocols vs. guidelines. Choosing a medical-dispatch program. Emerg Med Serv 1994, 23:52-60.

10. Ebben RH, Vloet LC, Verhofstad MH, Meijer S, Mintjes-de Groot JA, van Achterberg T: Adherence to guidelines and protocols in the prehospital and emergency care setting: a systematic review. Scand J Trauma Resusc Emerg Med 2013, 21:9.

11. Lorem T, Saether E, Wik L: Handling of drug-related emergendies: an evaluation of emergency medical dispatch. Eur J Emerg Med 2009, 16:37-42.

12. Zakariassen E, Burman RA, Hunskaar S: The epidemiology of medical emergency contacts outside hospitals in Norway-a prospective population based study. Scand J Trauma Resusc Emerg Med 2010, 18:9.

13. The Norwegian Directorate of Health: Nasjonale data Fra Akutmedisinske Kommunikasjonssentraler. Årsrapport 2012. (National data from Emergency Medical Communication Centers 2012) [http://helsedirektoratet.no/ publikasjoner/nasjonale-data-fra-akuttmedisinske-kommunikasjonssentralerarsrapport-2012]

14. Statistics Norway. [www.ssb.no]

15. National Centre on Emergency Communication in Health. [www.kokom.no]

16. Bakke HK, Hansen IS, Bendixen AB, Morild I, Lilleng PK, Wisborg T: Fatal injury as a function of rurality - a tale of two Norwegian counties. Scan J Trauma Resusc Emerg Med 2013, 21:14.

17. Vaardal B, Lossius HM, Steen PA, Johnsen R: Have the implementation of a new specialized emergency medical service influenced the pattern of general practitioners involvement in pre-hospital medical emergencies? A study of geographic variations in alerting, dispatch, and response. Emerg Med J 2005, 22:216-219.

18. Zakariassen $E$, Hunskaar S: Involvement in emergency situations by primary care doctors on-call in Norway-a prospective population-based observational study. BMC Emerg Med 2010, 10:5.

19. Andersen MS, Johnsen SP, Sørensen JN, Jepsen SB, Hansen JB, Christensen EF: Implementing a nationwide criteria-based emergency medical dispatch system: a register-based follow-up study. Scand J Trauma Resusc Emerg Med 2013, 21:53.

20. Zakariassen E, Hansen EH, Hunskaar S: Incidence of emergency contacts (red responses) to Norwegian emergency primary healthcare services in 2007-a prospective observational study. Scand J Trauma Resusc Emerg Med 2009, 17:30

21. Hinchey P, Myers B, Zalkin J, Lewis R, Garner D: Low acuity EMS dispatch criteria can reliably identify patients without high-acuity illness or injury. Prehosp Emerg Care 2007, 11:42-48.

22. Heward A, Damiani M, Hartley-Sharpe C: Does the use of the advanced medical priority dispatch system affect cardiac arrest detection? Emerg Med J 2004, 21:115-118.

23. Ramanujam P, Guluma KZ, Castillo EM, Chacon M, Jensen MB, Patel E Linnick W, Dunford JV: Accuracy of stroke recognition by emergency medical dispatchers and paramedics-San Diego experience. Prehosp Emerg Care 2008, 12:307-313.

24. Clawson J, Olola C, Heward A, Patterson B, Scott G: Ability of the medical priority dispatch system protocol to predict the acuity of "unknown problem" dispatch response levels. Prehosp Emerg Care 2008, 12:290-296.

25. Heward A: Dispatch research: Someone, somewhere needs to know. Ann Emerg Dispatch Response 2013, 1:5-8.

26. Ebben RHA, Vloet LCM, Schalk DMJ, Mintjes-de-Groot JAJA, van Achterberg $\mathrm{T}$ : An exploration of factors influencing ambulance and emergency nurses' protocol adherence in the Netherlands. J Emerg Nurs 2012, [Epub ahead of print].

doi:10.1186/1757-7241-22-2

Cite this article as: Ellensen et al:: Variations in contact patterns and dispatch guideline adherence between Norwegian emergency medical communication centres - a cross-sectional study. Scandinavian Journal of Trauma, Resuscitation and Emergency Medicine 2014 22:2.

\section{Submit your next manuscript to BioMed Central and take full advantage of:}

- Convenient online submission

- Thorough peer review

- No space constraints or color figure charges

- Immediate publication on acceptance

- Inclusion in PubMed, CAS, Scopus and Google Scholar

- Research which is freely available for redistribution 